Instituto Internacional de Investigación y Desarrollo Tecnológico Educativo INDTEC, C.A.

DOI: https://doi.org/10.29394/Scientific.issn.2542-2987.2020.5.17.4.79-99

OAI-PMH: http://www.indteca.com/ojs/index.php/Revista Scientific/oai

Artículo Original / Original Article

\title{
Fortalecimiento del pensamiento espacial-geométrico a través de las inteligencias múltiples en educación infantil
}

\author{
Autora: Deisy Yasmine González Rojas \\ Secretaría de Educación Distrital, SED \\ deisyyasmineg@gmail.com \\ Bogotá, Colombia \\ https://orcid.org/0000-0002-8667-9194
}

\section{Resumen}

Esta investigación-acción, se fundamentó en el propósito de fortalecer el pensamiento espacial-geométrico a través de las inteligencias múltiples, en estudiantes de educación infantil, en el colegio Miguel Antonio Caro IED, de Bogotá, entre los años académicos 2014 y 2018 respectivamente. La presente, se enmarcó en un enfoque mixto, de tipo descriptivo, en una población de escolares, de educación básica primaria, de grados tercero y quinto. La recolección de información se basó en la técnica de observación directa, en conjunción con los resultados logrados por los educandos, en las pruebas estatales SABER primaria y datos obtenidos con la aplicación del cuestionario del profesor, para diagnosticar inteligencias múltiples en primaria de Armstrong (2001); y la adaptación de Prieto y Ballester (2003). Estimados los datos perfilados, se concluyó, en la necesidad de mediar académicamente, por medio del diseño y ejecución de un programa de intervención pedagógica, estructurado con estrategias dinamizadas, desde las inteligencias múltiples, conducentes al afianzamiento del pensamiento espacial-geométrico, en los colegiales, lográndose potenciar habilidades y competencias matemáticas, propias del pensamiento espacial-geométrico.

Palabras clave: pensamiento; inteligencia; matemáticas; geometría; educación.

Cómo citar este artículo:
González, D. (2020). Fortalecimiento del pensamiento espacial-geométrico a través de las
inteligencias múltiples en educación infantil. Revista Scientific, 5(17), 79-99, e-ISSN: $2542-2987$.
Recuperado de: https://doi.org/10.29394/Scientific.issn.2542-2987.2020.5.17.4.79-99

Fecha de Recepción: 05-03-2020
Fecha de Aceptación: 09-06-2020
Fecha de Publicación: 05-08-2020 
OAI-PMH: http://www.indteca.com/ojs/index.php/Revista_Scientific/oai

Artículo Original / Original Article

\title{
Strengthening spatial-geometric thinking through multiple intelligences in early childhood education
}

\begin{abstract}
This action research was based on the purpose of strengthening spatialgeometric thinking through multiple intelligences, in early childhood students, at the Miguel Antonio Caro IED school, in Bogotá, between the academic years 2014 and 2018 respectively. The present one, was framed in a mixed approach, of descriptive type, in a population of schoolchildren, of primary education, of third and fifth grades. The collection of information was based on the direct observation technique, in conjunction with the results achieved by the students, on the state primary SABER tests and data obtained with the application of the teacher questionnaire, to diagnose multiple intelligences in Armstrong's primary school (2001); and the adaptation of Prieto and Ballester (2003). Estimated the outlined data, it was concluded, in the need to mediate academically, through the design and execution of a pedagogical intervention program, structured with dynamic strategies, from multiple intelligences, leading to the consolidation of spatial-geometric thinking, in schoolchildren , managing to enhance mathematical skills and competences, typical of spatialgeometric thinking.
\end{abstract}

Keywords: thinking; intelligence; mathematics; geometry; education.

\footnotetext{
How to cite this article:

González, D. (2020). Strengthening spatial-geometric thinking through multiple intelligences in early childhood education. Revista Scientific, 5(17), 79-99, e-ISSN: 2542-2987. Recovered from: https://doi.org/10.29394/Scientific.issn.2542-2987.2020.5.17.4.79-99
}

Date Received:

05-03-2020
Date Acceptance:

09-06-2020
Date Publication:

05-08-2020 


\section{Introducción}

Teniendo en cuenta que, desde hace tiempos inmemoriales, a la educación se le ha asignado la función de orientar y promover conocimientos, con la pretensión de estructurar aprendizajes significativos, productivos y que éstos, a su vez resulten ser transversales, además, de que también conlleven éstos al razonamiento analítico, en y durante los procesos sociales-educativos, surgió la inquietud pedagógica de considerar la mitigación y mejoramiento de falencias identificadas en el ejercicio cotidiano en el aula de clase, con escolares de Educación Básica Primaria o Infantil; de ahí que, el estudio investigativo, se efectuó en y desde la praxis escolar, en un colegio de carácter público, ubicado en la ciudad capital de Colombia, Bogotá D.C., desde el año lectivo 2014 y finalizada en el año académico 2018.

En consecuencia, la formación integral en los primeros años de vida escolar, requieren de un accionar pedagógico comprometido y secuencial, con miras a estimar las particularidades de los educandos, para así promover aprendizajes sólidos y significativos. A continuación, Mota (2016), expresa que: "[...] la educación primaria, se percibe como multicultural y cambiante, a lo que se añaden las diferencias personales de cada individuo en capacidades, situaciones transitorias o formas de entender la vida [...]" (pág. 141).

Por medio de, observaciones iniciales directas no estructuradas, en el año escolar 2014, resultados del cuestionario del profesor, para diagnosticar inteligencias múltiples en primaria de Armstrong (2001a); y la adaptación de Prieto y Ballester (2003a): y puntajes de las pruebas estatales SABER primaria, de los años lectivos 2015 y 2017, se dio inicio al diseño de un programa de intervención pedagógica que se cimentó, en el propósito del fortalecimiento del pensamiento espacial-geométrico, por medio de la dinamización de las inteligencias múltiples, en educación infantil, para grado quinto y tercero, implementado en los años académicos 2016 a grado quinto y luego adaptado para su ejecución con el grado tercero, en el año 2018. La 
OAI-PMH: http://www.indteca.com/ojs/index.php/Revista_Scientific/oai

\section{Artículo Original / Original Article}

intervención pedagógica, en la investigación acción, se fundamenta desde la reflexión del quehacer educativo cotidiano, observable, que pretendiendo innovar con acciones y/o estrategias, para lograr cambios favorecedores, acentúa la mirada, al contexto y a la diversidad de características particulares de la población a impactar. Posteriormente, Matos, Cegarra y Rivera (2017), sostienen que:

En consecuencia, partiendo de que el individuo como ser bio psico social cultural está inmerso en un contexto rodeado de personas con características muy particulares, el docente en su rol protagónico de socializador, pedagogo, didáctico, gerente y evaluador está constantemente interactuando tanto con el personal, como con sus estudiantes y la comunidad en sí (pág. 325).

El estudio realizado, es el producto del proyecto de investigación doctoral, denominado fortalecimiento del pensamiento espacial-geométrico, a través de las inteligencias múltiples en educación infantil, el cual se estimó a partir de la técnica de la observación directa de la necesidad notable de mejorar en dicho aspecto, las destrezas matemáticas, que resultaron evidentes en las prácticas docentes diarias, llevadas a cabo con los estudiantes de Educación Básica Primaria o infantil, de la jornada tarde, del colegio distrital Miguel Antonio Caro IED, en los grados quinto y tercero; lo anterior promovió la reflexión pedagógica, con miras al afianzamiento y aumento de las capacidades en procesos académicos-matemáticos, de los educandos, en grados de educación primaria o infantil, por tanto, se analizaron las posibles acciones para favorecer de manera particular, el pensamiento espacial-geométrico, en los colegiales de niveles básicos de formación escolar.

Aterrizando la mirada al aula de clase, desde el año lectivo 2014 donde la diversidad de casos y realidades por mejorar, se mostraban además de numerosos, heterogéneos y complejos, de variables dependientes e 
independientes, que intervinieron en el proceso educativo, se analizó la necesidad de mediar pedagógicamente, para integrar conocimientos, esbozar e implementar alternativas ejecutables, que contribuyeran a la solución y/o mejoramiento de la situación considerada para este momento, como problémica y como objeto del estudio investigativo, el cual se direccionó, al fortalecimiento del pensamiento espacial-geométrico, desde los grados iniciales de educación formal (tercero y quinto), entendiéndose para el estudio, de educación primaria o infantil.

En consecuencia, resultó particularmente interesante fijar la atención en situaciones que, para los estudiantes representaban dificultades académicas, adicionalmente, que éstas se encontraban estrechamente vinculadas con el desarrollo de procesos específicos de áreas fundamentales, como es el caso particular, del área de matemáticas. En el ejercicio docente en Educación Básica Primaria o infantil, se vislumbraron las incipientes falencias presentadas en los educandos frente a los avances, que se daban en la asimilación y desarrollo de los pensamientos matemáticos y sus sistemas constitutivos, como sucedió concretamente con el pensamiento espacial y los sistemas geométricos, pues éste además, de ser uno de los cinco pensamientos y sistemas, componentes de los estándares básicos de las competencias matemáticas, adaptados y adoptados por el Ministerio de Educación colombiano, desde hace unos años y que son los pilares en la fundamentación matemática aplicada.

Concretamente, el compendio temático del pensamiento espacialgeométrico carecía de relevancia en las prácticas escolares cotidianas, debido a que sus contenidos fijados y específicos, se habían dejado durante varios años lectivos, según las programaciones o mallas curriculares, para el final de los años escolares; situación que se notó, no permitía un trabajo acentuado y pertinente a las temáticas propias del ámbito espacial y geométrico. De manera tal que, dicha situación escolar generaba incongruencias, en muchos 
OAI-PMH: http://www.indteca.com/ojs/index.php/Revista_Scientific/oai

Artículo Original / Original Article

saberes básicos, útiles y necesarios, para la concatenación y formalización de aprendizajes esenciales, significativos, cardinales e indispensables en y para las asociaciones futuras de conceptos mayormente estructurados, en niveles educativos superiores, como sucede en la Educación Básica Secundaria.

De acuerdo con, la observación directa, individual y no estructurada, durante las prácticas académicas diarias y la implementación del programa de intervención pedagógica, diseñado y ejecutado para los niveles de quinto y tercero de Educación Primaria o infantil, en espacio escolar, se estimó dentro de su estructura, plasmar reflexiones y análisis de manera sistemática, relacionando actitudes logradas a partir de la interacción en tiempo escolar, con cada grupo de educandos. Notándose con las observaciones, que la mayoría de los escolares de Educación Primaria o infantil, presentaban escaso interés por las actividades matemáticas, adicionalmente, bajo desempeño académico, no solamente en las pruebas institucionales, sino también, en las pruebas estatales SABER primaria; se pretendió dinamizar las clases para buscar la mitigación principalmente del desánimo por el área académica.

Teniendo en cuenta las observaciones, en el año lectivo 2014, en los grados de quinto y tercero de Educación Primaria o infantil, en donde se notó entre otras, la alta mortalidad académica; para el año escolar 2015, se aplicó el cuestionario del profesor, para diagnosticar inteligencias múltiples en primaria de Armstrong (2001b); y la adaptación de Prieto y Ballester (2003b): a los educandos de quinto grado y se analizaron los resultados de la prueba estatal SABER primaria, de este grado escolar, hallando estadísticos de correlación entre los datos obtenidos del cuestionario del profesor, para diagnosticar inteligencias múltiples en primaria y los resultados de la prueba estatal.

Con la información de las observaciones y la correlación estadística, se procedió en el año académico 2016, a diseñar e implementar un programa de intervención pedagógica, en el grado quinto de Educación Primaria o infantil, 
con el propósito de coadyuvar en el fortalecimiento del pensamiento espacialgeométrico por medio de la potenciación de las inteligencias múltiples. Con los registros secuenciales y sistemáticos de las observaciones, durante el desarrollo de las estrategias diseñadas, se evidenció mayor interés por las clases y temáticas, asociadas al área de matemáticas y asignatura de geometría, por ende, se logró el fortalecimiento del pensamiento espacialgeométrico, de manera lúdica, creativa y en tiempo escolar.

De ahí que, para el año 2017 inmediatamente siguiente, se analizaron los resultados obtenidos en la prueba estatal SABER primaria, del grado tercero de Educación Primaria o infantil, a éste, se le aplicó el cuestionario del profesor, para diagnosticar inteligencias múltiples en primaria de Armstrong (2001c); y la adaptación de Prieto y Ballester (2003c): y se realizó la actividad de hallar la correlación estadística entre los datos conseguidos, de las pruebas y las inteligencias múltiples. Con las experiencias pedagógicas, la reflexión docente y la información obtenida, para el año lectivo 2018, se adaptó el programa de intervención pedagógica, que se había desarrollado con educandos de grado quinto, teniendo en cuenta las diversas caracterizaciones del grado tercero, como edad, gustos y demás particularidades y se ejecutó éste, con gran éxito, promoviendo incluso el gusto y deseo de aprender, a partir de la dinamización de las inteligencias múltiples, como es el caso de la interpersonal, muy eficaz ésta en el proceso de enseñanza-aprendizaje. Consecutivamente, Sánchez y Guedez (2017), afirman que:

Toda acción educativa debe estar basada en una comunicación interpersonal constante y efectiva entre todos los miembros del contexto escolar y dirigido hacia la búsqueda de una integración de esfuerzo para el desarrollo de planes, proyectos y programas que apunten al logro de objetivos propuestos (pág. 157).

El quehacer pedagógico, enmarcado en las acciones y/o estrategias, direccionadas en la escuela o centro educativo, en y durante los primeros 
grados de escolaridad, tendrá una gran influencia en los procesos de formación del educando. En tal sentido, Rodríguez, García y Fuentes (2020), afirman que: "[...] todo proceso de aprendizaje, desde los primeros años de vida, constituye un eje central en la formación del hombre como ser social e individual” (pág. 231).

El estudio se apoyó de primera mano, en la técnica de la observación directa, individual y no estructurada, al mismo tiempo de la descripción y explicación de las diferentes acciones que lo constituyeron, conducentes a la estimación de correlaciones.

\section{Metodología (Materiales y métodos)}

En un primer momento, tenidos los registros de las observaciones realizadas directamente a los educandos de grados quinto y tercero, de Educación Básica Primaria o infantil, de la jornada tarde, del colegio Miguel Antonio Caro IED y los respectivos análisis, tanto del ejercicio e interacción con y entre ellos, originados durante el desarrollo de las actividades académicas del área de matemáticas, como de las acciones ejecutadas en las clases, por los escolares, se pudo identificar y destacar, las siguientes dificultades: Consideraciones erradas de qué son y para qué son las matemáticas, falta de orientación en el espacio, mal empleo de elementos de trazo -regla, compás, transportador-, falta de interpretación de situaciones dadas, falta de manejo conceptual básico, para competencias del pensamiento espacial-geométrico.

En cuanto a las inexactitudes, en relación con las competencias matemáticas particularmente, del pensamiento espacial-geométrico, que fueron observadas y se reconocieron en el ejercicio del aula de clase y que, a través de la triangulación de datos, se llegó a la reflexión pedagógica que, de no tratarse, estas falencias evidenciadas en los niveles educativos primarios se podrían complejizar en grados superiores de educación académica formal 
y acarrear situaciones complejas, como Bajo rendimiento académico, apatía por el área, procesos matemáticos de lento desarrollo, deserción académica.

De ahí que, las falencias focalizadas, que se evidenciaron en el trabajo pedagógico diario con los escolares, requirieron de explicaciones reiteradas, de conceptos y de ejecución de planes de recuperación y/o nivelaciones permanentes que, además encaminaron en algunos estudiantes, el incremento en su falta de interés por el área de matemáticas y sus componentes, mortalidad académica y en algunos, hasta la deserción.

Por tanto, la descripción explicativa, del desarrollo del accionar pedagógico en contexto académico, se fortaleció por medio de la tabulación de las observaciones, que en principio se dieron de manera no estructurada y espontánea y que luego permitieron identificar falencias, presentadas durante el desarrollo de las actividades académicas desarrolladas en el aula de clase. Asimismo, el proceso de reflexión y autoevaluación docente coadyuvó en el proceso comprometido y de preocupación constante, que pretendió promover la concatenación de análisis de las dificultades presentadas, para estructurar y redireccionar estrategias implementadas y así, encaminarlas cada vez mejor, en la búsqueda de progresos significativos e incrementales, en los escolares.

En un segundo momento, con los datos obtenidos de los resultados de la prueba estatal SABER primaria y establecidas las valoraciones de ocho inteligencias múltiples en los escolares, por medio del cuestionario del profesor, para diagnosticar inteligencias múltiples en primaria, de Armstrong (2001d): se esbozó desde el enfoque cuantitativo, no experimental, teniendo en cuenta un esquema correlacional y descriptivo, a una muestra no aleatoria de sesenta y un estudiantes de grado quinto de la jornada tarde, del colegio Miguel Antonio Caro IED, un estudio investigativo en el que se refirieron individualizaciones relacionadas con el pensamiento espacial-geométrico y las inteligencias múltiples, de forma no experimental, debido a la poca o nula factibilidad de realizar controles a las variables. 
OAI-PMH: http://www.indteca.com/ojs/index.php/Revista_Scientific/oai

Artículo Original / Original Article

En un tercer momento, con las dinámicas observadas y registradas de las clases de matemáticas y el estudio correlacional-descriptivo, se diseñó un programa de intervención pedagógica con aportes de la neuropsicología y el pensamiento creativo, para el grado quinto de Educación Primaria o infantil, población de estudio que constó de sesenta y un escolares, implementándose éste en espacio y tiempo escolar. A través de las diversas acciones pedagógicas esbozadas y ejecutadas, con los educandos de grado quinto, en donde se introdujeron los recursos técnicos y tecnológicos existentes en el contexto inmediato, sus resultados y dinámica, generó la inquietud, por promover estrategias pedagógicas en el aula de clase con escolares de otro grado de Educación Primaria o infantil.

El diseño e implementación, del programa de intervención pedagógica de aula, estructurado para los estudiantes de grado quinto de Educación Primaria o infantil del colegio Miguel Antonio Caro IED, con el propósito de favorecer la asimilación de concepciones espaciales y geométricas, permitió la canalización de reflexiones educativas, generando cambios en la óptica de quienes fueron partícipes en el desarrollo de las acciones. El progreso en las prácticas pedagógicas dispuestas para el programa de intervención, en tiempo académico, permitió la identificación de tendencias y estilos de aprendizaje, los cuales direccionaron el enriquecimiento de las actividades programadas, por medio de la búsqueda de alternativas de dinamización y potencialización de los contenidos básicos del pensamiento espacial-geométrico y la aprehensión conceptual, de manera práctica en el aula de clase, con la activación de las diversas inteligencias múltiples.

Realizado el estudio investigativo, en el grado quinto de Educación Básica Primaria o infantil, se procedió al siguiente año académico, es decir, en el 2017, a realizar los análisis cualitativos y cuantitativos pertinentes, para replicar la investigación-acción, esta vez con estudiantes de tercer grado de Educación Primaria o infantil, teniendo en cuenta un esbozo no experimental, 
descriptivo y correlacional, a una muestra no aleatoria de treinta colegiales, refiriendo variables asociadas al pensamiento espacial-geométrico y las inteligencias múltiples, en éstos.

Con los resultados obtenidos del esbozo no experimental, cualitativo y cuantitativo, de las variables, en el grado quinto de Educación Primaria o infantil, se adaptó el programa de intervención pedagógica, con acciones educativas propias al contexto y caracterización particular de los escolares, para grado tercero. Se estimó, analizar observaciones realizadas a los educandos de grado tercero de Educación Primaria o infantil y llevar a cabo un estudio investigativo, no aleatorio inicial con este grado, teniendo presente la experiencia y los resultados, tanto del cuestionario para diagnosticar inteligencias múltiples en primaria, como de la prueba estatal SABER primaria del grado tercero, los cuales permitieron, en conjunción con las observaciones directas, una planeación y ejecución de estrategias educativas funcionales.

Por tanto, dadas las caracterizaciones de los grupos de estudio, se optó por implementar la Investigación-Acción (IA), ésta efectuada en el aula de clase, realizada con el propósito de aprovechar y dinamizar, conocimientos pedagógicos e investigativos, vinculados desde los aportes estadísticos de la correlación obtenida entre el pensamiento espacial-geométrico y las ocho inteligencias múltiples, valoradas en cada grupo de escolares.

De ahí que, la investigación se apoyó en los datos obtenidos, primeramente, de un estudio cuantitativo, no experimental, transformada luego en un trabajo de tipo cualitativo y experimental; con la finalidad de establecer y analizar la relación entre el pensamiento espacial-geométrico y las inteligencias múltiples, empleando un diseño correlacional, como punto de partida para la investigación-acción. Teniendo en cuenta un esbozo no experimental, correlacional y luego descriptivo, a una muestra no aleatoria, en la que se refirieron caracterizaciones relativas al pensamiento espacialgeométrico y a ocho inteligencias múltiples, de forma experimental, debido a 
OAI-PMH: http://www.indteca.com/ojs/index.php/Revista_Scientific/oai

Artículo Original / Original Article

la factibilidad en el desarrollo de acciones didácticas, diseñadas para un programa para intervención neuro-pedagógica, en espacio escolar, realizando controles a las variables.

En consecuencia, con los resultados obtenidos por el grado tercero y quinto, en las competencias matemáticas de la prueba SABER primaria, el registro de observaciones directas y las valoraciones del cuestionario de inteligencias múltiples (para infantil y primaria, aplicado para los dos cursos), los cuales presentaron puntuaciones bajas, se pensó en encaminar acciones pedagógicas, direccionando el diseño de un programa de intervención pedagógica, con fundamentaciones en neuropsicología, para estos cursos, de la jornada tarde, estimando las condiciones contextuales, los recursos de infraestructura y tecnológicos del entorno escolar, con miras a la ejecución, en tiempo y espacio escolar. Por consiguiente, se enteró a los padres de los estudiantes, del programa de intervención pedagógica a desarrollarse, por medio de un consentimiento informado.

El proyecto de Investigación-Acción, encaminado al fortalecimiento del pensamiento espacial-geométrico a través de las inteligencias múltiples en educación infantil, que se desarrolló en el colegio Miguel Antonio Caro IED, Bogotá, Colombia, se llevó a cabo desde la siguiente documentación de variables, para tener en cuenta en la investigación: pensamiento espacialgeométrico e inteligencias múltiples, estimando instrumentos de recolección de datos, la técnica de observación directa y el cuestionario del profesor para diagnosticar inteligencias múltiples en Infantil y Primaria. De manera que, se aprovechó como instrumento de estudio y análisis, el registro de observaciones realizadas en clase, en y durante el desarrollo de las actividades pedagógicas propuestas, al inicio del primer semestre de los años escolares 2014, 2015, 2016, 2017 y 2018, pretendiendo la búsqueda de estrategias a través de la reflexión pedagógica, para la mitigación de situaciones consideradas como falencias en las habilidades para desarrollar 
competencias matemáticas, específicamente inscritas al pensamiento espacial-geométrico; encaminando el accionar pedagógico e investigativo, a una investigación-acción, para fortalecer el pensamiento espacial-geométrico por medio de la dinamización de las inteligencias múltiples, en los educandos de Educación Básica Primaria o infantil.

En relación con, la ejecución del programa de intervención neuropedagógica que se llevó a cabo, en el espacio escolar del aula de clase, donde se realizó la ejecución de actividades tanto individuales como colectivas, con la intención de apuntalar y favorecer las habilidades y mitigar las debilidades encontradas, tanto en el manejo de aspectos espaciales, como geométricos, con la mediación de las diversas inteligencias, a través de procesos de creatividad, estimando ejecuciones concernientes al desarrollo de las cuatro fases del proceso creativo, que mencionan López-Fernández y LlamasSalguero (2018), que resultan ser: Preparación, incubación, iluminación y verificación, que promovieron la fijación y potenciación de cada competencia matemática; los resultados plasmados, son el producto de la triangulación de las observaciones realizadas a los estudiantes, los resultados de las pruebas SABER primaria y la implementación de las estrategias pedagógicas.

Por otra parte, teniendo presentes los registros secuenciales, de las actividades, componentes del programa de intervención docente, que como propósito principal tuvo, fortalecer el pensamiento espacial-geométrico, en los estudiantes de educación infantil, se diligenció el formato de registro sistemático de actividades, para cada una de las cuatro competencias matemáticas: comunicación, razonamiento, modelación y resolución de problemas. Como resultado, de las observaciones realizadas durante la implementación del trabajo investigativo, desde los aspectos funcional, estadístico y analítico, se llevó a cabo una reflexión del quehacer docente y su incidencia en la formación matemática de los escolares de Educación Básica Primaria, de la jornada tarde, del colegio distrital Miguel Antonio Caro IED. 
OAI-PMH: http://www.indteca.com/ojs/index.php/Revista_Scientific/oai

\section{Artículo Original / Original Article}

Entre tanto, resultó relevante también, la reflexión pedagógica asociada

a la educación como proceso social y dinámico, que se estructura y construye continuamente, dando así apertura al desarrollo holístico, en y para la vida. La realidad educativa, que se pudo intervenir, requirió del acopio de acciones direccionadas, desde el aula de clase en conjunción con la constante comprensión, de las diferentes dinámicas, que van más allá, del ejercicio diario y que, por consiguiente, demandan de un quehacer docente, analítico, abierto al cambio y de un espíritu investigativo, funcional e interviniente en los procesos que conforman la autenticidad escolar.

Por lo cual, estructurado e implementado el programa de intervención pedagógica, que tuvo como finalidad el fortalecimiento de habilidades propias del pensamiento espacial-geométrico, por medio de la ejecución de acciones activadoras de potencialidades múltiples, favoreció la potenciación de las competencias matemáticas, las cuales son: comunicación, razonamiento, modelación y resolución de problemas, coadyuvando en el propósito general del conjunto de actividades, conducentes a enriquecer el desarrollo de destrezas. Se realizó durante las acciones pedagógicas, un análisis de estrategias y/o actividades que se desarrollaran en el trabajo diario escolar y que, de manera diversa, lúdica y con ayuda de recursos técnicos y tecnológicos, en los cursos de tercero y quinto, de la jornada tarde, del colegio distrital Miguel Antonio Caro IED, en los años lectivos 2014, 2015, 2016, 2017 y 2018, acciones de observación más continua y acompañada de la reflexión docente.

En el desarrollo académico del año lectivo 2014, durante la ejecución de las labores académicas, se logró evidenciar situaciones relacionadas con la falta de habilidad y destreza, en el desarrollo de actividades inscritas de manera particular, con el pensamiento espacial-geométrico, básico para el constructo holístico y complejo de las conceptualizaciones matemáticas aplicadas a situaciones cotidianas del entorno inmediato y más amplio, de ahí 
que, en este año, se registraron las observaciones directas del accionar de los escolares, en y durante la ejecución de acciones académicas orientadas en las clases.

Teniendo como base, las experiencias y las observaciones registradas del año lectivo 2014, de actitudes en los estudiantes, consistentes de manera básica en la ejecución de actividades propiamente relacionadas con el área de matemáticas (asignatura de geometría), para el año lectivo 2015, se consideró pertinente llevar a cabo una investigación-acción, por lo que además de las observaciones, se empleó el cuestionario del profesor, para diagnosticar inteligencias múltiples, a los escolares de grado quinto, en conjunción con los resultados de éste, se tuvo en cuenta las valoraciones obtenidas en la prueba SABER primaria, del grado quinto, para hallar las correlaciones estadísticas, entre las variables de pensamiento espacial-geométrico y las inteligencias múltiples, y así mediar, a través de las de mayor puntuación, acciones que enriquecieran y a la vez promocionaran aprendizajes, que permitieran la dinamización e innovación de conceptualizaciones cada vez más y mejormente esbozadas.

Subsiguientemente, a las observaciones directas en el ejercicio diario de las clases, en el espacio escolar del aula de clase, los resultados del cuestionario para diagnóstico de inteligencias múltiples, los resultados obtenidos en las pruebas SABER primaria, la determinación de las correlaciones calculadas estadísticamente, entre el pensamiento espacialgeométrico y las inteligencias múltiples y la reflexión pedagógica, acerca de lo efectuado desde el año 2014, para el año lectivo 2016, se diseñó un programa de intervención pedagógica, implementado en el aula de clase, en tiempo escolar, con estrategias del pensamiento creativo y estimulando desde las diversas inteligencias, destrezas que favorecieron también, la realización de observaciones directas del desempeño y de la participación de los educandos durante el desarrollo de las actividades propuestas, orientadas a potenciar el 
OAI-PMH: http://www.indteca.com/ojs/index.php/Revista_Scientific/oai

Artículo Original / Original Article

pensamiento espacial-geométrico, lográndose cambios interesantes, especialmente en la actitud de interés y aceptación de los educandos por efectuar actividades, coadyuvantes al mejoramiento del desempeño matemático, desde el pensamiento creativo, permitiéndose disfrutar del trabajo académico en tiempo de clase.

En conjunción con, los registros de las observaciones sistemáticas y los cambios logrados en los educandos de grado quinto, con el desarrollo de las estrategias pedagógicas planificadas para fortalecer el pensamiento espacialgeométrico a través de las inteligencias múltiples, en éstos escolares, se procedió en el año lectivo 2017, a focalizar el estudio de investigación-acción, para los estudiantes del grado tercero de Educación Básica Primaria, de la jornada tarde, del colegio distrital Miguel Antonio Caro IED, por tanto, se tomó como base los registros de las observaciones no estructuradas y directas, que se habían realizado en el año lectivo 2014, asociados a las valoraciones obtenidas del cuestionario para diagnosticar inteligencias múltiples en primaria, aplicado a éstos colegiales y los resultados de la prueba estatal SABER primaria de este grado, con la finalidad de, adaptar e implementar el programa de intervención pedagógica, buscando favorecer el mejoramiento de falencias en el pensamiento espacial-geométrico, en este grado.

\section{Resultados (análisis e interpretación de los resultados)}

Con los datos y resultados tenidos, de las variables independientes, cuantitativas continuas, del pensamiento espacial-geométrico y las inteligencias múltiples, en asociación con el análisis correlacional entre éstas, se estructuró un diseño y se llevó a cabo la implementación de un programa de intervención pedagógica, con apoyo de conceptualizaciones del pensamiento creativo, en las actividades conformantes y estratégicas, que hicieron parte integral de la investigación-acción, que se fundamentó en el objetivo inicial, de analizar la relación existente entre el pensamiento espacial- 
geométrico y las inteligencias múltiples, en un grupo de noventa y un estudiantes de los grados tercero y quinto de Educación Básica Primaria, por medio de los estadísticos de correlación de Pearson, para comprobar hipótesis planteadas, en relación a las habilidades y destrezas, espaciales y geométricas.

La interacción continua con los educandos favoreció el constructo de una serie de ejercicios modeladores que orientaron las temáticas del pensamiento espacial-geométrico en éstos los grados quinto y tercero de Educación Primaria, reflexionando constate y permanentemente sobre la importancia de las matemáticas en diversos contextos de nuestra la cotidianidad. Se evidenció una progresión incremental en la curiosidad e interés de los escolares, durante el trabajo realizado, a partir de sus inquietudes y participación en el desarrollo de las acciones propuestas. Aunque no se logró que la totalidad de los estudiantes presentaran un nivel alto de comprensión, durante la ejecución de las actividades realizadas, si se generó un ambiente de confianza y reflexión que permitirá en un futuro próximo, la asociación de saberes más complejos del pensamiento espacialgeométrico y en general de las matemáticas.

Gráfico 1. Apreciación referente a las clases de matemáticas.

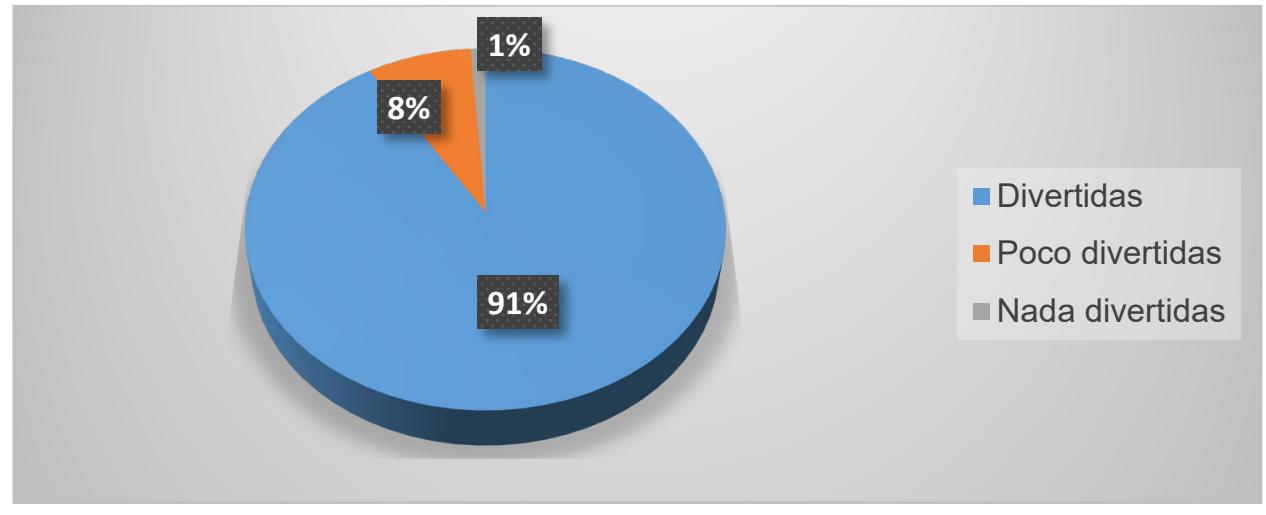

Fuente: La Autora (2020). 
OAI-PMH: http://www.indteca.com/ojs/index.php/Revista_Scientific/oai

\section{Artículo Original / Original Article}

Los resultados de la investigación-acción, que se realizó desde el año 2014, hasta el año 2018, en el colegio distrital Miguel Antonio Caro IED de la ciudad de Bogotá D.C., por parte de los escolares de grado quinto y tercero de Educación Primaria o infantil, de la jornada tarde, mostraron que la mayoría de las actividades en la clase de matemáticas, eran divertidas en un $91 \%$ y que podían también aprender, no solamente copiando y/o transcribiendo en sus cuadernos del tablero, como se aprecia en el gráfico 1.

Como resultado, el diseño y ejecución de la estrategia pedagógica de aula, para los estudiantes de grado quinto y tercero de Educación Primaria del colegio Miguel Antonio Caro, jornada tarde, que proyectó favorecer el avance en la maduración y enriquecimiento del pensamiento espacial-geométrico, orientando con su realización, el constructo de actividades próximas que posibilitaran mejoramientos en la adquisición de los saberes y la gestión de los mismos en condicionamientos contextuales propios, promovió también una mejor y mayor interacción entre compañeros/as.

\section{Conclusiones}

Conviene subrayar, que la observación directa de los comportamientos que adoptan los estudiantes, durante la operatividad de las acciones contempladas en el programa de intervención pedagógica, coadyuva en el proceso de evaluación de las acciones mismas, posibilitando su análisis de manera continua y constante. La programación y desempeño de labores pedagógicas en el aula, es una herramienta que posibilita la generación de intervalos para la reflexión, adicionalmente demanda del docente un conocimiento y experiencia en la didáctica del área de matemáticas, para entrelazar tanto las temáticas, con los contenidos y esto en conjunto con los atributos particulares del contexto inmediato, teniendo en cuenta la caracterización de la población escolar, de tal manera que es conminatorio proyectar actividades prácticas, que dinamicen los conceptos y cooperen en 
la significación de conceptos.

De manera tal que, el trabajo geométrico sencillo y práctico en el aula de clase, proporciona recursos valiosos al proceso de aprendizaje, de todos los participantes en el mismo (docente, estudiantes), de tal manera que en relación a los escolares, se beneficia la potencialización de los conceptos básicos del pensamiento espacial-geométrico, favoreciendo la comprensión de su importancia en la cotidianidad; mientras que para el/la docente, se convierte en un canal de aprehensión metacognitivo, que posibilita la realización de cambios e innovaciones en la realidad de cada uno de los escenarios didácticos próximos. Es decir, que la investigación cualitativaInvestigación-Acción, incrementa la preparación y el intelecto profesional, a partir de la estimulación de diversas habilidades, direccionadas a la reflexión crítica.

Por tanto, las prácticas impulsadas durante el quehacer pedagógico favorecen la interacción con y entre los participantes del ejercicio investigativo, que han de enriquecer el proceso integral formativo, de los escolares y del/a docente. Los aspectos técnicos y metodológicos de la investigación cualitativa direccionan y optimizan el quehacer investigativo en la educación; la comprensión de conceptos y la construcción de conocimientos, se favorece y perfecciona desde la labor investigativa, con acciones coherentes y de pertinencia para el contexto inmediato que secunden procesos aún más complejos en la transformación de ambientes, a través de la conducción de cambios desde los espacios escolares.

En cuanto a la ejecución de la investigación-acción, la intervención como agente investigador/a, se considera un medio para la reflexión continua y constante, partiendo del compromiso misional de orientar procesos significantes, en las vidas de los/as escolares, mejorando y/o activando exponencialmente sus habilidades inquisitivas propias, del ámbito pedagógico actual, en la sociedad del conocimiento. 


\section{Referencias}

Armstrong, T. (2001a,b,c,d). Inteligencias Múltiples: cómo descubrirlas y estimularlas en sus hijos. ISBN: 9580464081. San José, Costa Rica: Grupo Editorial Norma.

López-Fernández, V., \& Llamas-Salguero, F. (2018). Neuropsicología del proceso creativo. Un enfoque educativo. Revista Complutense de Educación, 29(1), 113-127, e-ISSN: 1988-2793. Recuperado de: https://doi.org/10.5209/RCED.52103

Matos, Y., Cegarra, O., \& Rivera, C. (2017). La Praxis Docente desde la Formación Permanente. Revista Scientific, 2(4), 319-336, e-ISSN: 2542-2987. Recuperado de:

https://doi.org/10.29394/scientific.issn.2542-2987.2017.2.4.18.319-336

Mota, J. (2016). Supervisión Vs Calidad Educativa en Educación Primaria. Revista Scientific, 1(2), 131-146, e-ISSN: 2542-2987. Recuperado de: https://doi.org/10.29394/scientific.issn.2542-2987.2016.1.2.8.131-146

Prieto, M., \& Ballester, P. (2003a,b,c). Las inteligencias múltiples: Diferentes formas de enseñar y aprender. ISBN: 84-368-1820-2. España: Ediciones Pirámide.

Rodríguez, M., García, W., \& Fuentes, C. (2020). Valores éticos y emociones desde el desarrollo de metodologías activas en la formación docente. Revista Scientific, 5(15), 229-246, e-ISSN: 2542-2987. Recuperado de: https://doi.org/10.29394/Scientific.issn.25422987.2020.5.15.11.229-246

Sánchez, H., \& Guedez, A. (2017). La Comunicación Interpersonal y el Proceso Formativo Creativo en la Escuela Primaria Venezolana. (Caso: Escuela "Palenque Abajo", Buena Vista, Venezuela). Revista Scientific, 2(Ed. Esp.), 154-173, e-ISSN: 2542-2987. Recuperado de: https://doi.org/10.29394/scientific.issn.2542-2987.2017.0.0.9.154-173 


\section{Deisy Yasmine González Rojas}

e-mail: deisyyasmineg@gmail.com

Nacida en Colombia, Departamento de Cundinamarca,

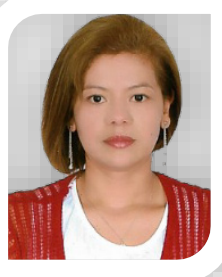
municipio de Nocaima, el 15 de abril de 1981. Doctora en Educación; Magister en Neuropsicología y Educación; Magister en Docencia Universitaria; Licenciada en Educación y Promoción de la Comunidad, con énfasis en Matemáticas; Bachiller Pedagógica; Me he desempeñado por más de veinte años, en el campo de la educación, como docente de educación preescolar, primaria y secundaria, como directiva docente en el sector rural y urbano, con comunidades escolares, de todas las edades. 